\title{
ISME activity on the use of Autonomous Surface and Underwater Vehicles for acoustic surveys at sea
}

\author{
Gianluca Antonelli ${ }^{1}$, Filippo Arrichiello ${ }^{1}$, Andrea Caiti ${ }^{2}$, Giuseppe Casalino ${ }^{3}$, Daniela De Palma ${ }^{4}$, \\ Giovanni Indiveri ${ }^{4}$, Matteo Razzanelli ${ }^{2}$, Lorenzo Pollini ${ }^{2}$, Enrico Simetti ${ }^{3}$ \\ ${ }^{1}$ ISME-node University of Cassino and Southern Lazio, Italy \\ ${ }^{2}$ ISME-node University of Pisa, Italy \\ ${ }^{3}$ ISME-node University of Genova, Italy \\ ${ }^{4}$ ISME-node University of Salento, Italy
}

\section{ABSTRACT}

The paper presents an overview of the recent and ongoing research activities of the Italian Interuniversity Center on Integrated Systems for the Marine Environment (ISME) in the field of geotechnical seismic surveying. Such activities, performed in the framework of the H2020 European project WiMUST, include the development of technologies and algorithms for Autonomous Surface Crafts and Autonomous Underwater Vehicles to perform geotechnical seismic surveying by means of a team of robots towing streamers equipped with acoustic sensors.

\section{Section: RESEARCH PAPER}

Keywords: WiMUST

Citation: Gianluca Antonelli, Filippo Arrichiello, Andrea Caiti, Giuseppe Casalino, Daniela De Palma, Giovanni Indiveri, Matteo Razzanelli, Lorenzo Pollini, Enrico Simetti, ISME activity on the use of Autonomous Surface and Underwater Vehicles for acoustic surveys at sea, Acta IMEKO, vol. 7, no. 2, article 5, June 2018, identifier: IMEKO-ACTA-07 (2018)-02-05

Section Editor: Fabio Leccese, Università degli Studi di Roma Tre, Italy

Received January 15, 2018; In final form March 22, 2018; Published June 2018

Copyright: @ 2018 IMEKO. This is an open-access article distributed under the terms of the Creative Commons Attribution 3.0 License, which permits unrestricted use, distribution, and reproduction in any medium, provided the original author and source are credited

Funding: This work has received funding from the European Union's Horizon 2020 research and innovation programme under the grant agreement No. 645141 (WiMUST project)

Corresponding author: Filippo Arrichiello, e-mail: f.arrichiello@unicas.it

\section{INTRODUCTION}

ISME, the Interuniversity Center on Integrated Systems for the Marine Environment, is an Italian Institution that involves a set of Universities (named as ISME-Nodes) that actively work in the field of marine robotics and acoustic systems. ISME benefits from the research infrastructure of the participating departments (laboratories, workshops, etc.), and it has the purpose of exploiting synergies and collaborative efforts among the participants within an agile and flexible organizational structure. In the recent years, ISME has been involved in several National and European Projects concerning acoustics and marine robotics, as e.g. [1], [2]. The full list of projects can be found on the internet portal: http://www.isme.unige.it

Among its recent activities, ISME has been involved in different research projects concerning the development and the use of Autonomous Surface Crafts (ASCs) and Autonomous
Underwater Vehicles (AUVs) for a number of applications including environmental monitoring and seismic surveys. In particular, ISME has coordinated the H2020 Research and Innovation Action funded by the European Commission (Work Programme 2014 - 2015, LEIT- ICT, 5. Leadership in enabling and industrial technologies - Information and Communication Technologies) named Widely scalable Mobile Underwater Sonar Technology (WiMUST), 2015-2018. http://www.wimust.eu/

The main objective of the WiMUST project was the design, development, and testing of a robotic system composed of cooperating ASCs and AUVs to perform acoustic based geotechnical surveying and geophysical exploration [3]-[5]. To this purpose, the WiMUST project involved four academic partners and five industrial partners with specific expertise in the field of marine robotics, acoustic systems, and geophysical and geotechnical surveys: ISME, Instituto Superior Técnico 
(IST), Centro de Investigação Tecnológica do Algarve (CINTAL), University of Hertfordshire (UH), Graal Tech s.r.l. (GT), EvoLogics (EL), CGG, Geo Marine Survey Systems (GEO), and Geo Surveys (GS).

Acoustic seismic surveying methods allow the exploration of the sea bottom and sea bottom subsurface for applications that span from the geophysical domain (e.g. oil \& gas) to the geotechnical one (e.g. civil and commercial applications, underwater constructions). The seismic acoustic survey is traditionally performed using a surface vessel carrying one or more powerful acoustic sources (e.g. sparkers or airguns), and towing a number of streamers equipped with several hydrophones to record acoustic signals. Sparkers generate seismic waves by intermittently releasing electric pulses that produce low frequency sound waves. Such acoustic waves travel towards the sea floor and are reflected back to the hydrophones. The time delay with which waves return to the surface, registered with the hydrophones, provides valuable information about the properties of the ocean sub-bottom.

The WiMUST project aims at expanding and improving the functionalities of current cooperative marine robotic systems by effectively enabling a distributed acoustic array technology for seismic surveying. Indeed, the WiMUST system is composed of two ASCs carrying a sparker each, and of a fleet of AUVs towing streamers equipped with hydrophones to acquire subbottom profiling acoustic data. The hydrophones on the streamers towed by the AUVs can be envisioned as a $3 \mathrm{D}$ acoustic array that, by means of the autonomous and coordinated motion among ASCs and AUVs, can have an adaptive variable geometry. Indeed, by actively controlling the geometry of the robot formation, it becomes possible to change the shape of the acoustic array antenna, according to the needs of the considered application (see Figure 1).

The resulting operational flexibility holds potential advantages, as it allows improving the seabed and sub-bottom resolution and obtaining sidelobe rejection at almost any frequency and for any plane. The availability of the proposed system, other than possibly improving the quality of the acquired data, also greatly facilitates the operations at sea, thanks to the lack of physical ties between a surface ship and the acquisition equipment. On the other side, the lack of the towed streamers from the surface vessel introduces high complexity due to the need of ad-hoc cooperative navigation and control architecture for the team of ASCs and AUVs, as well as the establishment of an underwater acoustic communication network, where all the nodes need to be synchronized [6]. Moreover, the hydrophones' positions,

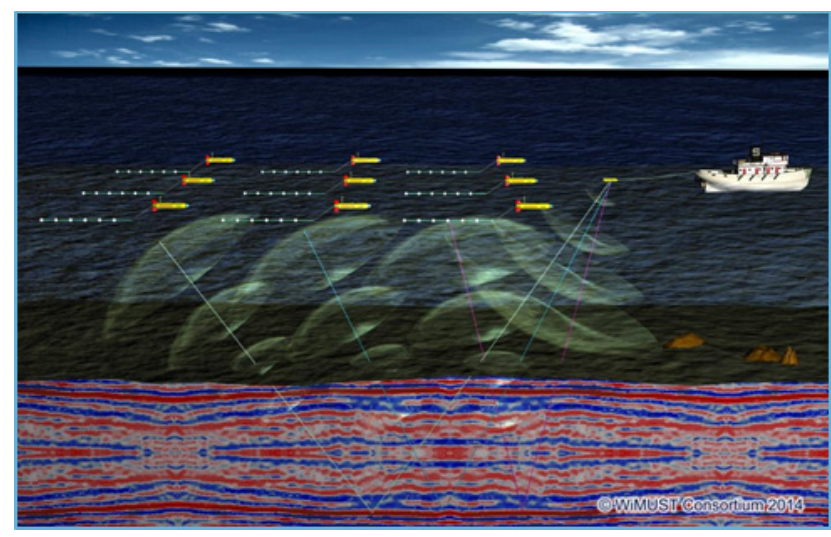

Figure 1. Sketch of the WiMUST system. needed for acoustic data processing, cannot be easily computed as in the case of surface surveys since the AUVs while diving cannot rely on GPS.

\section{ISME ACTIVITIES IN THE WIMUST PROJECT}

Among the overall activities of the WiMUST project, the contributions of ISME (nodes of Lecce, Genova, Pisa and Cassino) can be identified in the following activities.

\subsection{System integration}

ISME was responsible for the overall system integration of the WiMUST project that involves software and hardware integration of the different components of the system, i.e. the different type of autonomous vehicles (Delfim catamaran from IST, ULISSE catamaran from ISME, 4 Medusa AUVs [7] from IST, 4 Folaga AUVs from ISME and Graaltech), the acoustic communication devices, the streamers with hydrophones and the sparkers. Thus, the system integration deals with issues as: mechatronic integration of the sensing payloads and the communication devices on board the AUVs; software integration of the modules concerning group navigation and coordination; software integration of communication protocols and strategies to manage two acoustic communication networks (medium frequency for acoustic localization and high frequency for seismic data quality control), and a wi-fi network for the communication on the sea surface; hardware and software integration of the sparkers with the ASC catamarans. The overall system has been validated through experimental tests at sea (three integration weeks have been already performed in Sines, Portugal in November 2016 and July 2017, and in Lisbon in December 2017). For the final experiments, performed in January 2018, the autonomous vehicles constituting the WiMUST system have performed cooperative guidance, navigation, and control by implementing the solutions and methods developed for the project purposes and to acquire geoseismic acoustic data of a specific area. The overall WiMUST control architecture allowed the vehicles to successfully perform maintain formation while concurrently performing operational tasks related to the level of individual power supply, intra-vehicle distance, and quality of service of vehicle-to-vehicle communication.

To manage the fleet of AUVs and ASCs composing the WiMUST system, a range based localization strategy and a formation control algorithm have been developed by IST to make the system navigate in preassigned formation (see Figure 2). In this context, ISME activities has been focused on the development of low level motion control strategies for the ULISSE catamaran and for the Folaga AUVs towing streamers.

\subsection{Development of an Autonomous Catamaran}

The ISME-node Genova has designed and realized the ULISSE platform, i.e. the second generation of autonomous

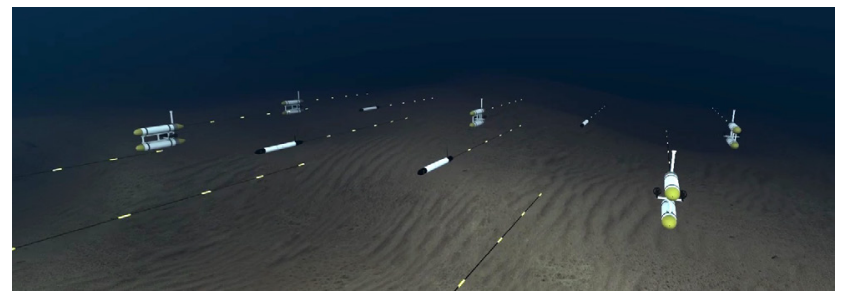

Figure 2. Computer animation of a WiMUST mission towing streamers Ulisse ISME Autonomous Catamaran. 
surface vehicles, following a previous design created for a private company in 2015. ULISSE is a $3 \mathrm{~m}$ long and $1.8 \mathrm{~m}$ wide catamaran, built in fiberglass (see. Figures 3-5). The two hulls are fixed together thanks to two carbon fiber bars, which pass through the two hulls below the deck line, and are fixed on both the interior and exterior of the catamaran. The catamaran has been designed capable to transport payloads up to $200 \mathrm{~kg}$.

The catamaran is propelled by two commercial Torqeedo Cruise $2 \mathrm{R}$ electric motors, of $2 \mathrm{~kW}$ electrical power and about $50 \%$ propulsive efficiency. The motor control electronics is connected to the ULISSE control electronics through a RS485 bus line. The motors can run in reverse, producing around 50\% of the thrust w.r.t. going forward. This allows the ASC to be controlled in differential mode, although some scaling needs to be taken into account to handle the different forward/reverse

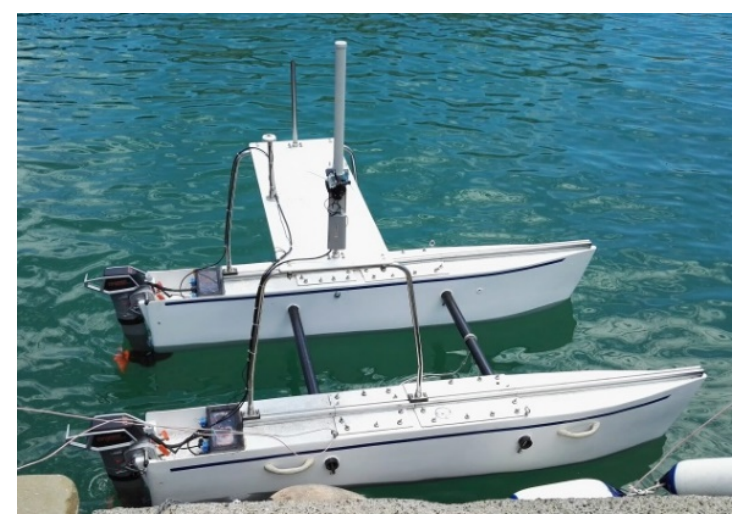

Figure 3. ULISSE with no payload mounted.

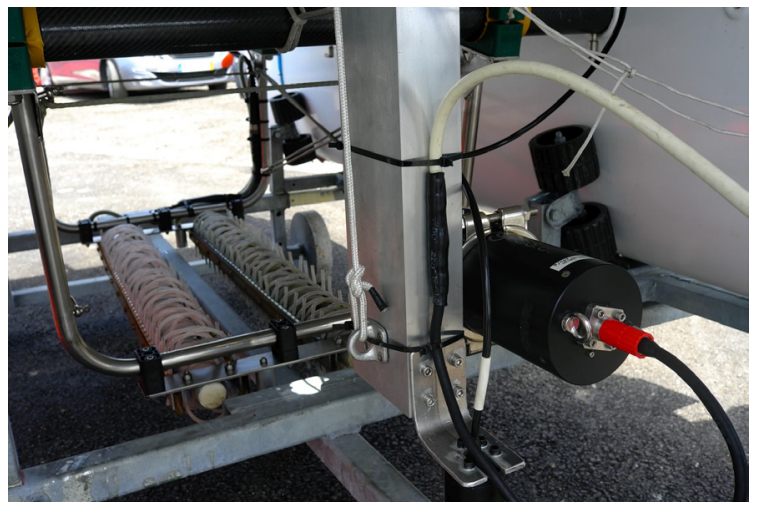

Figure 4. ULISSE configuration for the WiMUST system: the acoustic sparker and the two acoustic modem transducers can be seen in the middle part and front part respectively.

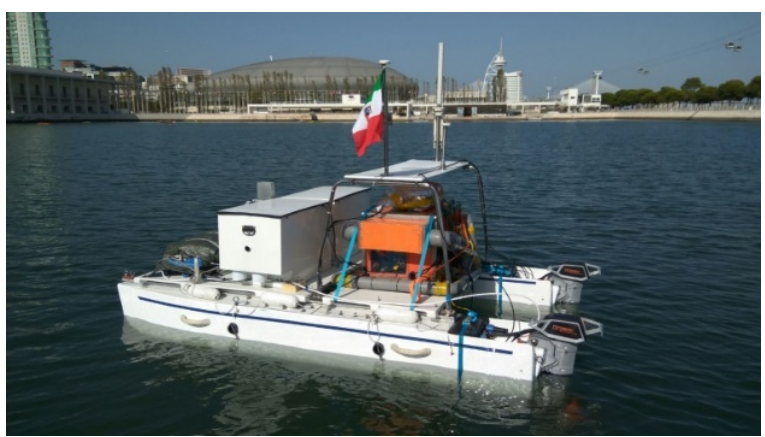

Figure 5. Ulisse ISME Autonomous Catamaran. thrust properly.

The vehicle is controlled by a PCM-3362 single board computer, equipped with a $1.67 \mathrm{GHz}$ Intel ${ }^{\circledR}$ Atom $^{\text {TM }}$ N450 processor. While the computational power is moderate, it is however sufficient for running path following and low level control algorithms needed for the WiMUST survey.

Both hulls have four internal compartments. The first one, accessible through a waterproof hatch, hosts the control electronics and the battery modules (around $3.2 \mathrm{kWh}$ energy each). The starboard side one also hosts the PCM single board computer, the inertial sensors (compass, accelerometers and gyroscopes), as well as the batteries). The second and third compartments, one near the bow and one near the stern, host two ballast tanks, which are controlled by pumps and electrovalves contained in the fourth compartment. The ballast system can be used to adjust the nominal roll and pitch of the vehicle once a certain payload has been installed.

The vehicle has been designed with the possibility of maximum re-configurability. In fact, along both hulls there is an aluminum Bosch Rexroth profile, which can be used to fix custom decks hosting the necessary payload for the mission at hand. Motors cables go through a connector box that is placed on the stern side of both hulls. These boxes are connected together, to ensure that all the electronics is connected to the control computer that is installed in the starboard hull. Furthermore, an Ethernet cable connects the control computer to an Ubiquiti $5 \mathrm{GHz}$ access point, located on the roll-bar. A further Ethernet connector is available to connect a payload to the internal network. Finally, both connector boxes host keys to turn on and off the system, a connector to recharge the battery and a spare power connection that can be used to power up payloads, with about25A@24V power available.

Communication with the base station or with other surface vessels is granted by a standard $5 \mathrm{GHz}$ communication system, which proved to be sufficient for the distances that were so far tested (around $300 \mathrm{~m}$ ). The first engineering tests have shown that the vehicle was capable of a speed of around $4 \mathrm{~m} / \mathrm{s}$ (about 8 knots) when the propellers were commanded at about $75 \%$. It is therefore estimated that the maximum achievable speed is around 10 knots. Furthermore, the two motors allow for a differential control of the catamaran and thus the vessel can turn on the spot. The maximum rotational speed on the spot is around $25 \mathrm{deg} / \mathrm{s}$.

In the WiMUST project, ULISSE carries one of the two acoustic sparkers. The sparker itself has been mounted with a clamp system, exploiting the two carbon fiber bars, in the middle part of the vehicle, in such a way to be at least $30 \mathrm{~cm}$ below the water level. The sparker can be seen in Figure 4. The sparker is powered by a power supply that charges high-voltage capacitors $(4 \mathrm{kV})$ necessary to create the spark.

Two dedicated decks have been manufactured for the WiMUST project. One, installed near the stern under the rollbar hosting the antennas, was dedicated to hosting the sparker's power supply. The second one, installed near the bow, hosts the movable pole to lower down the modem's transducers, an additional waterproof case hosting the modems electronics, and two Honda EU20i portable power generators, necessary to produce the $220 \mathrm{~V}$ for the sparker's power supply. Still in Figure 4, the two modems transducers (one for seismic data communication and one for acoustic localization) can be seen near the vehicle's bow.

The bow deck also hosts an additional waterproof case, containing the modem electronics and an Ethernet switch, 
which connects the two modems and the sparker triggering electronics to the onboard network and to the outgoing $5 \mathrm{GHz}$ connection.

The catamaran is capable of autonomously navigating exploiting information from onboard sensors, as GPS, IMU, compass, and of the information exchanged with other network nodes using Wi-Fi for surface nodes and acoustic modems for underwater nodes.

The motion control software of the ULISSE catamaran has been enhanced with a control mode, in the framework of ROS (Robot Operating System) [8], composed of a heading controller and a surge controller. Figures 6-10 show the results of a mission execution where the ASC was commanded to follow a trajectory specifically designed for the survey. In particular, Figure 6 shows the ASC position as measured from GPS signal. Figures 7-8 respectively show the ASC actual/desired heading and the error of the heading control

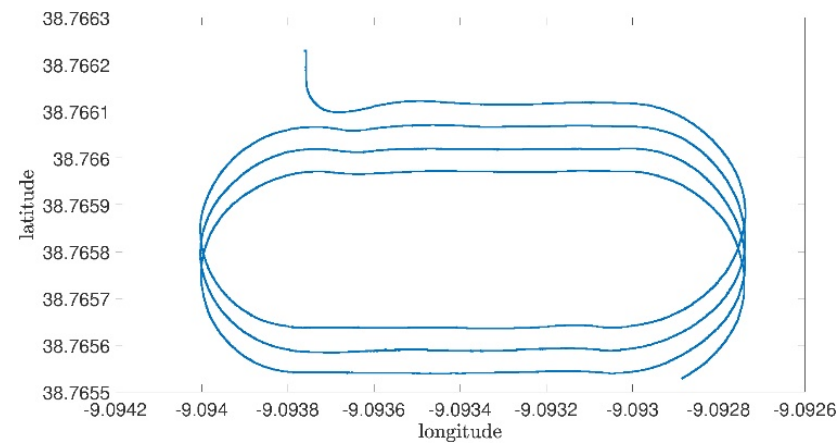

Figure 6. Autonomous navigation mission with Ulisse catamaran: effective trajectory reconstructed from GPS data.

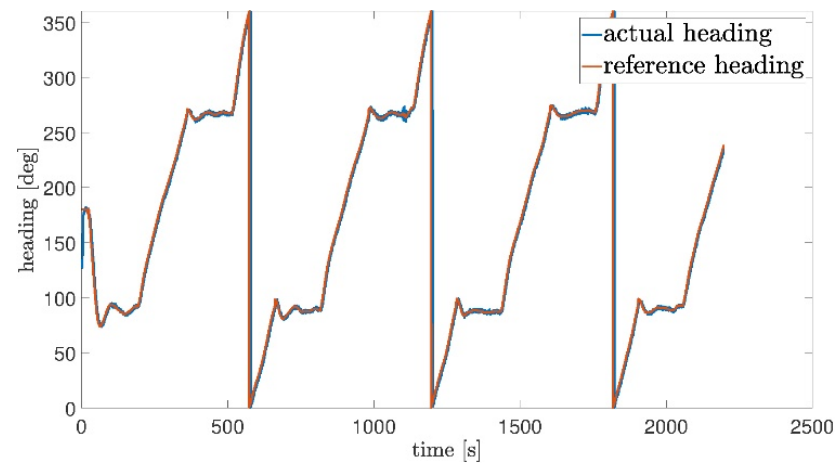

Figure 7. Autonomous navigation mission with Ulisse catamaran: reference and actual heading.

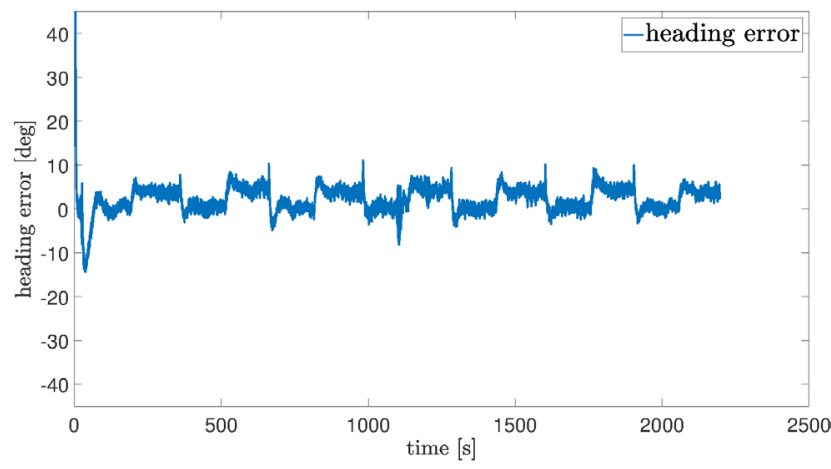

Figure 8. Autonomous navigation mission with Ulisse catamaran: error of the heading control.

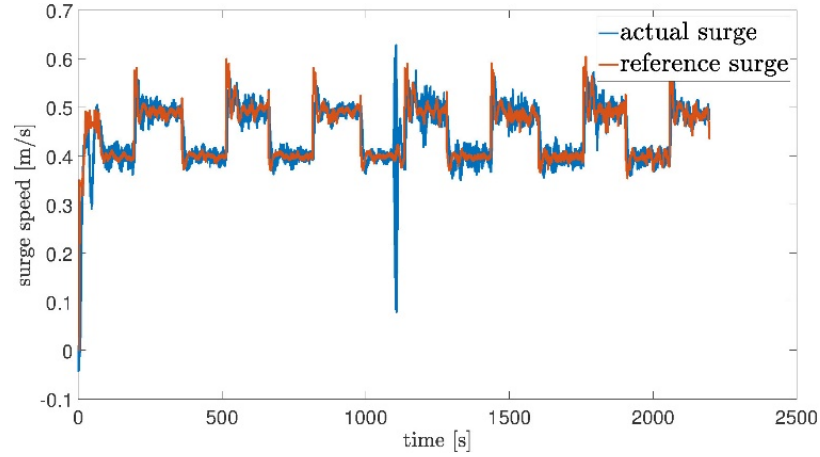

Figure 9. Autonomous navigation mission with Ulisse catamaran: reference and actual surge.

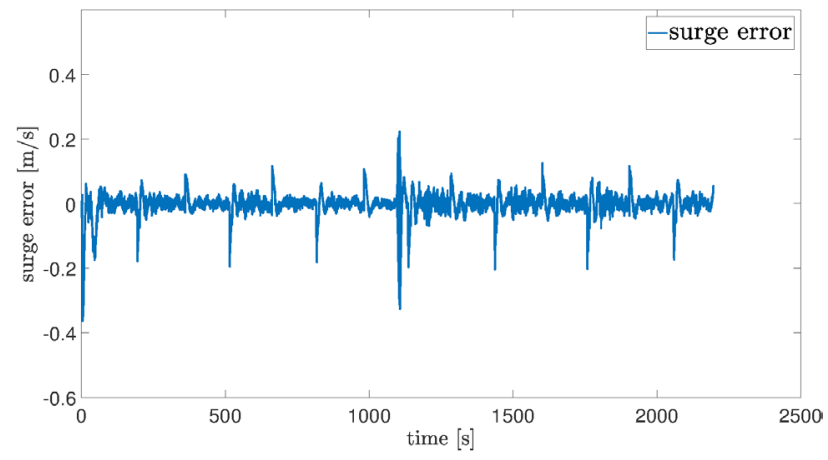

Figure 10. Autonomous navigation mission with Ulisse catamaran: error of the surge control.

algorithm (a PD controller). Figures 9-10 respectively show the ASC actual/desired surge and the error of the surge control algorithm (a PI controller).

\subsection{Folaga AUV motion control}

The Folagas are torpedo-like AUVs developed from Graal Tech Srl. The vehicle is actuated by 8 waterjet pumps and 1 propeller. The propeller provides trust along the surge direction, while 8 pumps, 4 in the front and 4 at the rear, generate forces and torques on the vehicle that allow lateral and vertical translation, and pitch and yaw rotation. The internal battery pack is mounted on a motorized frame that allows to change the longitudinal position of the AUV's center of mass. The Folaga AUV hull has a modular structure that allows adding one or more segments containing payload instrumentations. Within the WiMUST a specific payload module has been realized containing a single board computer, two acoustic modems, the electronic boards for signal conditioning and data storage of seismic data and the mechanical interface for the streamer, the array of hydrophones that constitutes the main mission payload. The streamer, about 10 meters long and towed by the AUV, collects seismic signals reflected by the sea bottom in order to analyze its geophysical structure; Figure 11 shows 4 Folagas with their streamers laid on the dock.

The Folaga AUV has a built in mission control system that allows performing basic operations. Early tests in Lisbon and Sines (2016) highlighted that the existing Folaga mission control system could not be used for implementation of a complete WiMUST mission where precise trajectory tracking and coordination with other vehicles is necessary. Thus, a new mission control system was overlaid to the existing one in order 


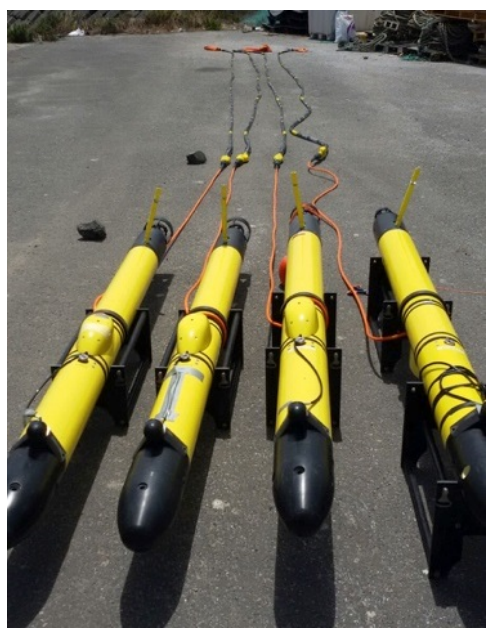

Figure 11. Folaga AUVs towing streamers with hydrophones.

to implement the necessary functionalities. The new control system was implemented in the additional single board computer (in the payload segment) using the Robot Operating System (ROS). The use of ROS, as middleware for control system implementation, revealed successful in particular with respect to the ease of integration of the control modules that were developed by the different partners of the project: The structure of the control system is shown in Figure 12.

The new Folaga mission control system is composed by several different Guidance, Navigation, and Control modules. Reference depth for the mission is set prior to mission beginning, but can be changed during the mission using a specific acoustic message that the vehicle receives trough the acoustic modems; this allows reconfiguration of the mission formation during its execution. The Depth controller is a fuzzyPID control system that blends two different control approaches: when the vehicle velocity is low, vertical pumps are used to control depth (with the vehicle almost leveled). When velocity is higher instead, the vehicle pitch angle is used as control variable to increase or decrease depth; this latter is the control mode that is used mostly throughout the mission. The adoption of this technique was necessary since the velocity of the vehicles during the mission may vary, and the effectiveness of depth control using the pitch angle is poor when velocity is low. The fuzzy blending strategy was tuned with field tests. Velocity and Heading controllers are of PID-type, and the Control Allocation block, specific for the pumps geometry, takes care of assigning appropriate pump and propeller commands in order to achieve the desired thrust and control

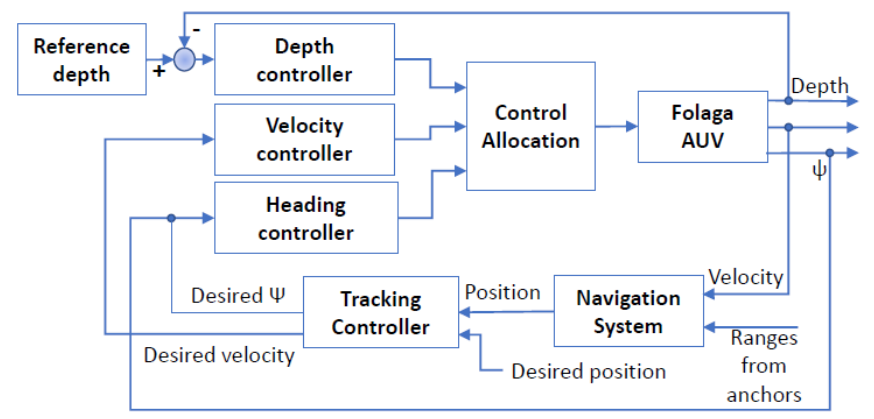

Figure 12.1 Block Diagram of the Folaga Guidance, Navigation and Control system. forces and moments. The navigation system used when the vehicle is at the surface fuses vehicle odometry and GPS measurements using a Kalman Filter (KF). A different navigation system, developed by IST, is used when underwater. The underwater Navigation system is composed of a Kalman Filter and a Maximum Likelihood Estimator (MLE), developed by IST. These two, together, use measurements of ranges between the vehicle and a set of anchors to estimate the vehicle position when underwater. The anchors are equipped with acoustic modems that ping periodically sending current anchor position and time. Ping times are synchronized using the GPS Pulse-Per-Second (PPS) signal; estimation of distance is performed by measuring the time of flight of acoustic signals and using Chip-scale Atomic Clocks (CSAC) inside the modems allows keeping a common time base with minimal drifts during the entire duration of the mission. The vehicles after reception of the pings from the anchors, calculate their distance from them and use this information to update the position estimate in the Kalman filter. At least two anchors are needed to compute a position fix; in the WiMUST architecture, surface vehicles (the two catamarans usually) act as anchors.

Thanks to the adoption of ROS as middleware, the KF and MLE components were implemented as two ROS nodes that run unmodified on the Folagas and the Medusas, even if the two AUVs differ both in hardware and software. The MLE node requires an estimate of vehicle velocity as input; since no DVL is present on the Folagas, a static mapping between propeller RPMs and vehicle velocity was created through extensive tests performed in La Spezia (Italy).

The Guidance system is implemented by the Tracking Controller ROS node, developed by IST and University of Hertfordshire (UH). The Tracking Controller computes feasible reference velocity and heading angle for the AUVs, and the catamarans in order to guide the vehicle from their initial positions toward the mission starting point prior to the start of the survey, and to remain in a fixed relative position with respect to the formation leader during the survey. In addition, an observer [9] is employed to estimate sea current in order to achieve a very precise formation tracking.

Again, thanks to the adoption of ROS, a complete virtual environment was created where entire missions were simulated prior to sea trials in order to verify correctness of the integration of the various software components. Then, sea trials, initially in La Spezia, later in Lisbon and Sines, were performed to field test the integration and to tune the Tracking Controller parameters to the Folaga dynamics.

Sea trials in La Spezia, Lisbon and Sines were performed to field test the integration and to assess the Folaga vehicle capability to remain in formation while underwater.

Figures 13-15 illustrate the results of a 2 hours experimental test involving, a virtual ship and 3 actual vehicles. The virtual ship, simulated by a PC in ground control station, follows a racetrack like trajectory several times and acts as formation leader. Two Folagas, vehicles F_57 and F_55 in the figure, were configured to stay in formation with the virtual ship, and to remain at the surface. The two surface Folagas were also configured to act as localization anchors, that is their acoustic modems were set up to ping periodically and send AUV position estimated using the onboard surface navigation system. The third Folaga, F_54 was configured as underwater vehicle, that is it used range measurements to F_57 and F_55 (obtained from the acoustic modem) to compute its position while navigating underwater. In particular, Figure 13 shows the 


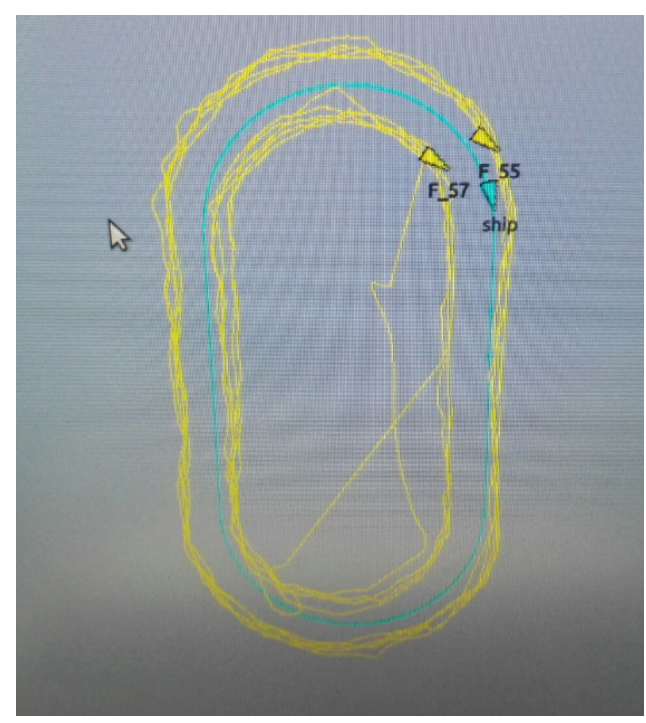

Figure 13. Results of an experimental test with two Folaga AUVs navigating at the surface and following a virtual ship leader.

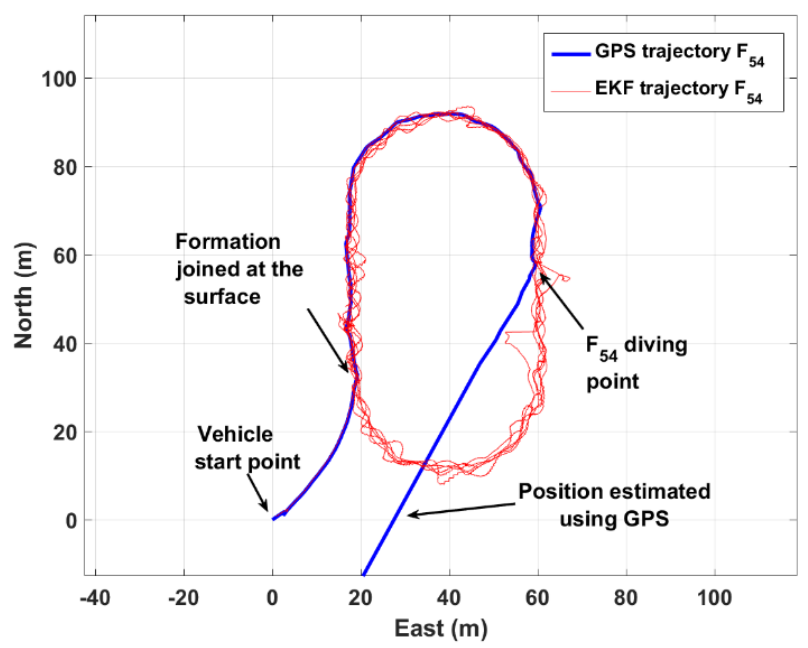

Figure 14. Results of an experimental test with a Folaga estimating its position while diving using range measurements from two surface nodes.

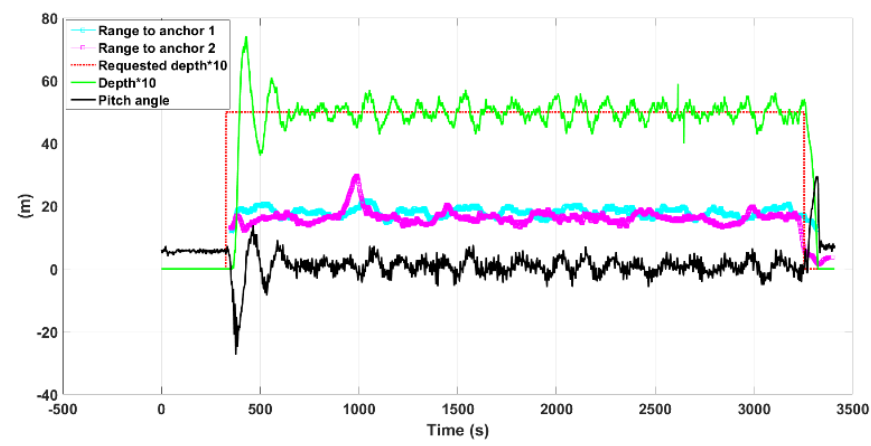

Figure 15. Results of an experimental test with a Folaga: depth, pitch and acoustic ranges measurements.

trajectory of the formation leader (the virtual ship), and of the surface Folagas as shown in the ground control station console screen developed by GT. The two large apparent deviations from the desired trajectory of F_55 and F_57 are actually artifacts part of the initial vehicle maneuvers needed to reach formation with the ship starting from their initial positions.

Figure 14 shows the trajectory of Folaga F_54; part of the mission was conducted at the surface: F_54 started from its initial position, joined the formation and conducted half of the racetrack at the surface before receiving the command to dive. Then the vehicle dives to 5 meters depth, and starts using the IST navigation system output (EKF trajectory) as feedback for the tracking Controller. The output of the navigation system used at the surface, which relies on GPS, drifts soon after the vehicle dives.

Figure 15 shows desired and measured depth during the mission, the AUV pitch angle, and the ranges to the anchor nodes.

\subsection{Hydrophones' localization algorithm}

For a proper post-processing of the WiMUST acquired acoustic data, there is the need to know the hydrophones' positioning during the navigation; however, the absence of a localization infrastructure requires the development of a strategy to estimate the hydrophones' positioning from the available set of information.

To the purpose, the ISME-node Cassino developed a localization strategy for the AUV-streamer system that, beyond using the data from navigation sensors of the AUV, as gyroscope, compass, Inertial Measurement Unit, USBL (as in [10]), it also makes use of information gathered from acoustic signals generated for the seismic survey. Indeed, considering that the sparkers and AUVs are equipped with acoustic modems with atomic clocks that allow precise synchronization, it is possible to use the direct time of arrival of the acoustic signals from the sparker to the hydrophones to compute range measurements. Thus, in the proposed localization strategy, range measurements from sparker to each hydrophone (see Figure 16) are collected and fused with AUV on board navigation sensors to estimate the overall system state using an Extended Kalman Filter.

To the purpose, the dynamic model of the AUV-streamer system (see Figures 17-18) has been firstly derived considering

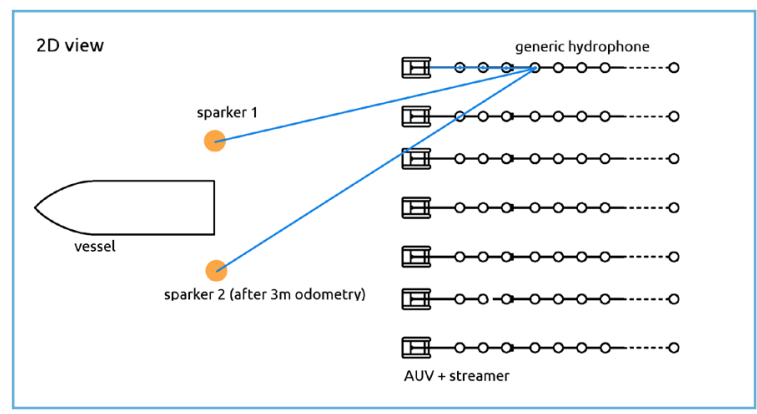

Figure 16. Sketch of the localization problem.

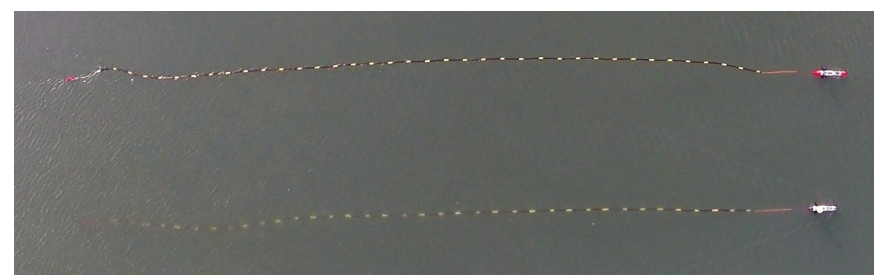

Figure 17. Picture of Medusa AUVs towing a streamer with hydrophones. 


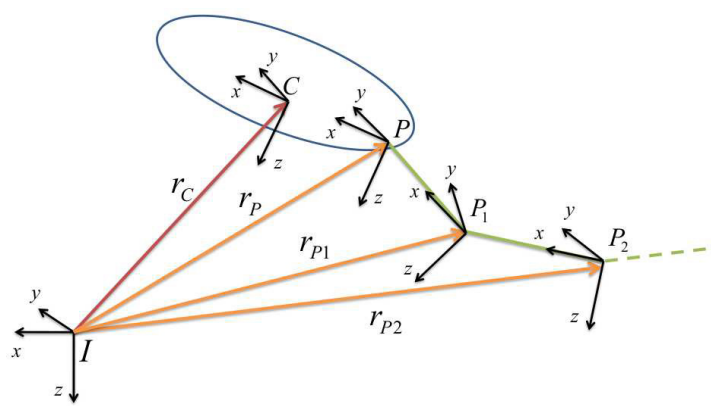

Figure 18. Modelling of AUV-streamer system.

the AUV as a fully-actuated rigid body, and the streamer as a serial chain of rigid links with spherical unactuated joints in correspondence of each hydrophone as [11].

The underwater dynamics of each rigid body has been modelled following the approach in [12]. In particular, the parameters of the dynamic model of the AUV have been defined on the basis of the Folaga characteristics, i.e. a $2 \mathrm{~m}$ length torpedo shaped AUV; the streamer, instead, has been modeled as a set of almost neutrally buoyant thin cylindrical elements connected via unactuated spherical joints. Nominal hydrodynamic terms and drag coefficients have been chosen accordingly to the effective geometry of the system, and on the basis of the common velocity regimes of the WiMUST system (i.e. cruise velocity about $1 \mathrm{~m} / \mathrm{s}$ ). Given the large dimension of the overall system state, the direct dynamic functions, needed for both numerical simulations of the system dynamics and for the computation of the prediction terms of an Extended Kalman Filter, have been developed referring to the NewtonEuler recursive procedure used for industrial manipulators [13].

The overall localization approach has been extensively tested in numerical simulations in Matlab environment, considering the case of single and multiple sparkers, and considering the AUV equipped with different set of navigation sensors. Moreover, the localization algorithm has been tested using data gathered from the experimental tests performed for the WiMUST project purposes (Sines, Portugal, Nov. 2016). A preliminary study on the feasibility of the localization strategy of the AUV-streamer system considering the availability of range measurement from sparkers to hydrophones has been investigated in [14]; however, that study was based on the use of an instantaneous set of sensor data, assuming the system as static. We then extend such work by developing the system dynamic model (in [15]), and by deriving the EKF equations and performing numerical validations also integrating data from experimental tests.

The acoustic signals collected with the hydrophones in the experimental campaigns were stored in the format of SEG-Y files, i.e. the standard format for storing geophysical data. Specifically, the SEG-Y file contains a set of traces for each hydrophone, where a trace represents the trend of the acoustic signal in a time slot of predefined length and time synchronized with the acoustic signal generated by the sparker. By post processing the SEG-Y file, beyond studying the sea subsurface from the signal reflections, it was also possible to extract the range measurements from the sparkers to hydrophones by computing the direct time of arrival of the acoustic signals (i.e. the time to reach the hydrophone without reflections on the sea bottom). Figure 19 illustrates an example of the range data extracted from the SEG-Y file and filtered out of a set of outliers following a threshold base approach.

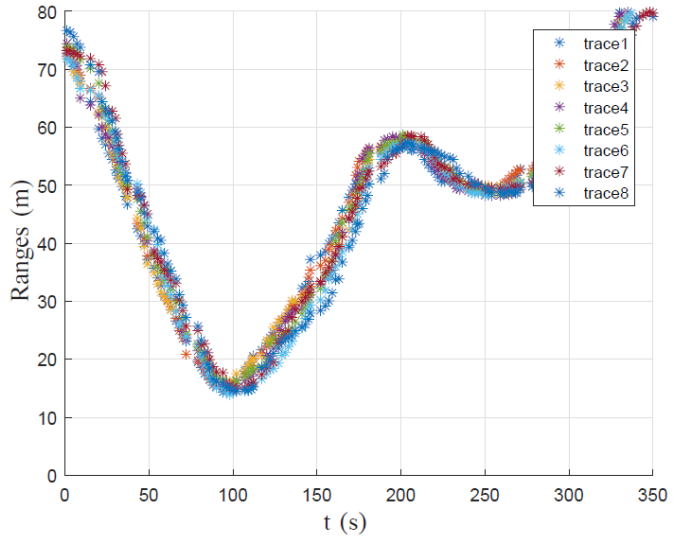

Figure 19. Range measurements from the sparker to the hydrophones collected from geoseismic acoustic data.

Such data have been used, together with the AUV's navigation sensor measurements, to perform a realistic simulation of the hydrophones' localization approach performance. In that tests, the AUV was commanded to navigate on the surface, so GPS readings were available and used as desired reference trajectory for a simulated AUV, where a basic PI controller has been used as a low-level dynamic control law.

Figure 20 shows the actual and the estimated position of the AUV and of the hydrophones while the AUV followed the desired path. The norm of the positioning estimation error for each hydrophone is plotted in Figure 21. From the figure it can

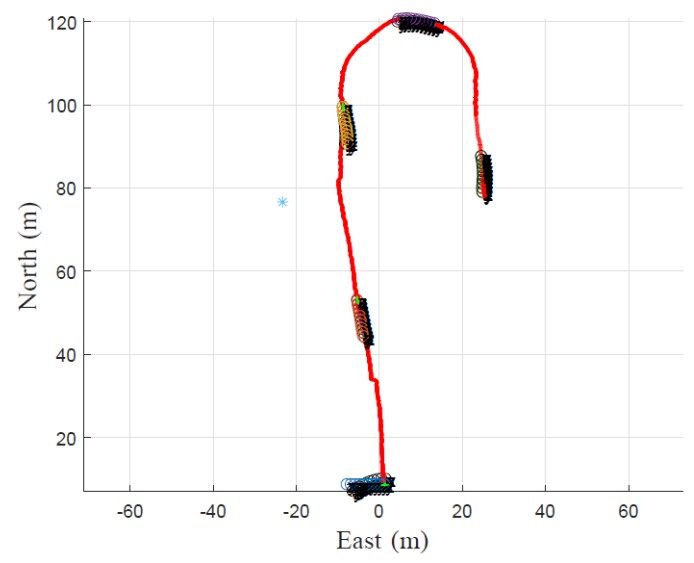

Figure 20. Plot of the desired trajectory (red), and few snapshots showing the AUV and hydrophones real and estimated positions.

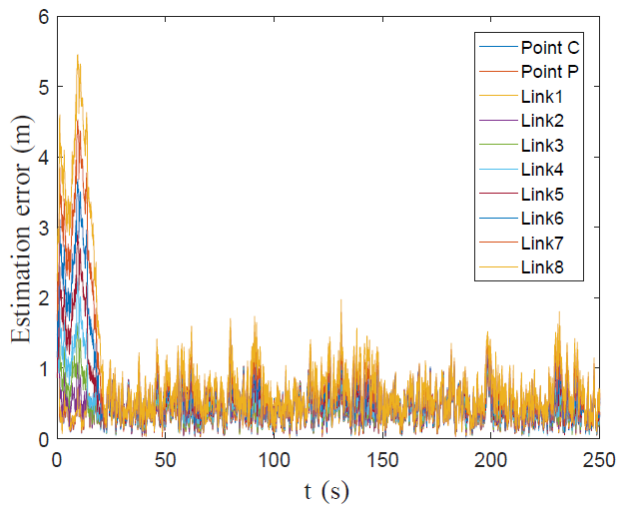

Figure 21. Plot of the hydrophones estimation errors. 
be noticed that, as can be expected, the estimation error decreases from the large initial error value, it keeps relatively low values with oscillations due to the process and measurement noise, and it keeps highest values for the farthest hydrophone from the AUV.

\section{CONCLUSIONS}

In this paper we presented an overview of the activities of the ISME Interuniversity Center performed for the H2020 WiMUST project (under grant agreement N. 645141), and concerning the development of a team of marine robots to perform acoustic seismic survey. In particular, the ISME activity has focused on the complete design of an Autonomous Catamaran to carry a sparker, on the development of control strategies for a the Folaga Autonomous Underwater Vehicles, on the coordination of the system integration activities of the project, and on the localization of hydrophone positioning using range measurement from seismic signals.

\section{REFERENCES}

[1] Casalino, G., Allotta, B., Antonelli, G., Caiti, A., Conte, G., Indiveri, G., Melchiorri, C., Simetti, E., "ISME research trends: Marine robotics for emergencies at sea". In OCEANS 2016Shanghai (pp. 1-5). IEEE, 2016

[2] Di Lillo, P. Simetti, E., De Palma, D., Cataldi, E., Indiveri, G., Antonelli G., Casalino, G., "Advanced ROV autonomy for efficient remote control in the DexROV project". Marine Technology Society Journal, 50(4), 67-80, 2016

[3] Al-Khatib, H., Antonelli, G., Caffaz, A., Caiti, A., Casalino, G., de Jong, I.B., Duarte, H., Indiveri, G., Jesus, S., Kebkal, K., Pascoal, A., and Polani, D. (2015b). "Navigation, guidance and control of underwater vehicles within the widely scalable mobile underwater sonar technology project: an overview". In 4th IFAC Workshop on Navigation, Guidance and Control of Underwater Vehicles NGCUV 2015 Dedicated to the memory of Professor Geoff. Roberts, volume 48, 189-193

[4] Al-Khatib, H., Antonelli, G., Caffaz, A., Caiti, A., Casalino, G., de Jong, I.B., Duarte, H., Indiveri, G., Jesus, S., Kebkal, K., Pascoal, A., and Polani, D. (2015a). "The widely scalable mobile underwater sonar technology (WiMUST) project: an overview." In Proceedings of MTS/IEEE Oceans '15, 1-5. Genova, Italy.
[5] Antonelli, G., Arrichiello, F., Caffaz, A., Caiti, A., Casalino, G., Volpi, N.C., de Jong, I.B., De Palma, D., Duarte, H., Gomes, J.P., Grimsdale, J., Indiveri, G., Jesus, S., Kebkal, K., Kelholt, E., Pascoal, A., Polani, D., Pollini, L., Simetti, E., and Turetta, A.. "Widely scalable mobile underwater sonar technology: an overview of the H2020 WiMUST project." Marine Technology Society Journal (Research Initiatives in Europe: Cooperation for Blue Growth), 50(4). Guest Editors: Andrea Caiti, Giuseppe Casalino and Andrea Trucco., vol. 50,n.4, pp. 42--53, 2016

[6] Valls, Oriol Pallares, Pierre-Jean Bouvet, and Joaquín del Río. "Hybrid time synchronization for Underwater Sensor Networks." ACTA IMEKO 4.3 (2015): 30-35.

[7] Abreu, P., Botelho, J., Gois, P., Pascoal, A., Ribeiro, J., Ribeiro, M., Rufino, M., Sebastiao, L., and Silva, H. (2016). The MEDUSA class of autonomous marine vehicles and their role in EU projects. In Proc. OCEANS 2016 MTS/IEEE - Shanghai, China, 10-13 April.

[8] Quigley, Morgan, et al. "ROS: an open-source Robot Operating rosSystem." ICRA workshop on open source software. Vol. 3. No. 3.2. 2009.

[9] Aicardi, M., Casalino, G., Indiveri, G., Aguiar, A., Encarnaçao, P., \& Pascoal, A. (2001, June). A planar path following controller for underactuated marine vehicles. In Ninth IEEE Mediterranean Conference on Control and Automation, Dubrovnik, Croatia

[10] F. Arrichiello, H. K. Heidarsson, and G. S. Sukhatme, "Opportunistic localization of underwater robots using drifters and boats," in 2012 IEEE International Conference on Robotics and Automation, May 2012, pp. 5307-5314 .

[11] J. E. Cochran, M. Innocenti, T. S. No, and A. Thukral, "Dynamics and control of maneuverable towed flight vehicles," Journal of Guidance, Control, and Dynamics, vol. 15, no. 5, pp. 1245-1252, Sep 1992.

[12] T. Fossen, Marine Control Systems: Guidance, Navigation and Control of Ships, Rigs and Underwater Vehicles. Trondheim, Norway: Marine Cybernetics, 2002

[13] B. Siciliano, L. Sciavicco, L. Villani, and G. Oriolo, Robotics: Modelling,Planning and Control. Springer, 2009.

[14] F. Arrichiello, G. Antonelli, and E. Kelholt, "Shape estimate of a streamer of hydrophones towed by an autonomous underwater vehicle," IFAC-PapersOnLine, vol. 49, no. 23, pp. 181 - 186, 2016

[15] F. Arrichiello, S. Sarkar, S. Chiaverini, and G. Antonelli, "Dynamic modelling of a streamer of hydrophones towed with an autonomous underwater vehicle," Modelling and Simulation for Autonomous Systems. MESAS 2017. Lecture Notes in Computer Science, vol 10756. Springer, Cham, pp 179-192, 2018. 